\title{
Light Trapping Enhancement in Thin Film Silicon Solar Cell with Different Back Reflector
}

\author{
Raghvendra Sarvjeet Dubey, Saravanan Sigamani*
}

Department of Nanotechnology, Swarnandhra College of Engineering \& Technology, West Godavari, India

Email address:

nanotechhod@swarnandhra.ac.in (R. S. Dubey), shasa86@gmail.com (S. Saravanan)

${ }^{*}$ Corresponding author

\section{To cite this article:}

Raghvendra Sarvjeet Dubey, Saravanan Sigamani. Light Trapping Enhancement in Thin Film Silicon Solar Cell with Different Back Reflector. International Journal of Electrical Components and Energy Conversion. Vol. 3, No. 5, 2017, pp. 83-87.

doi: $10.11648 /$ j.ijecec.20170305.11

Received: December 26, 2017; Accepted: January 10, 2018; Published: January 22, 2018

\begin{abstract}
Enhanced conversion efficiency with reduced cost of thin film solar cell is the primary objective of the scientific community. Light trapping mechanism is essential which have been explored and improved the performance of the solar cell. Past few decades, the plasmonic solar cell has been investigated in which actively involves metal nanostructure like nanoparticle or grating. Our numerical work analyzes the light absorption enhancement in the $1 \mu \mathrm{m}$ thin c-Si solar cell with different nanostructures. The optimal design combines front dielectric (ITO) and back metal (Ag) gratings as the reflector. This designed cell shows enhanced absorption through localized surface plasmon (LSP) or surface plasmon resonance (SPR). Comparing reference, the dual grating solar cell provides a significant efficiency from $\sim 11 \%$ to $18.82 \%$, which is a $71 \%$ enhancement. This enhancement has been attributed to the field localization and resonantly field scattering at the interface of the metal and active region.
\end{abstract}

Keywords: Conversion Efficiency, Thin Film, Light Trapping, Surface Plasmon Polariton

\section{Introduction}

The improvement of conversion efficiency and low cost thin film solar cell is the objective for the researchers. The efficient light trapping mechanism in the thin film solar cell becomes the key role to obtaining low cost and high efficiency solar cell as possible for commercial production. An earlier period, many light trapping techniques have been investigated to analyze the solar cell applications. For example, micron size pyramidal surface texture is the example of light trapping techniques in the past years [1-2]. One of the major drawbacks is its fairly low absorptances for photons within longer wavelength region, because low absorption coefficient [3]. Nowadays, to magnify the light trapping efficiency using different approach such as different back reflectors [4-5], designing periodic arrays of metal nanoparticles [6-7], modulating surface textures, twodimensional photonic crystal, diffraction grating (GRA), localized surface plasmon (LSP) and photonic crystal back reflector, etc. The absorption enhancement occurs due to strong field localization and resonantly enhance field scattering near the metal-absorbing material interface. The LSP in metal nano-gratings has been widely studied as a promising way to enhance light absorption in thin film solar cells. However, most research groups have been investigating dielectric and metal nanograting is separately. Here, three solar cell designs are proposed which an ultrathin film covered by periodic array of Ag and ITO nano gratings and compared with reference and metal back reflector structure.

The light trapping has been influenced by the shape and dimension of the GRA structure, in this light trapping under the variable of nano-gratings. The gratings can vary in following ways: (1) the gratings are placed different layers within the solar cells, (2) changing the gratings thickness (tg), (3) by change of the shape of the grating cells, and (4) by enhanced the symmetry of the photonic crystal grating etc. The top grating utilizes the scattering effects to allow more light coming into the solar cell to improve the overall trapping efficiency. The bottom silver $(\mathrm{Ag})$ back reflector grating is used to reflect and scattered light, which was not absorbed during the light trapping through the solar cell. The rigorous coupled wave analysis (RCWA) method is 
implemented to calculate optical performance of the designed solar cell with specified spectral range (300-1200nm) under AM1.5 solar radiation. The transverse electric and magnetic field polarizations are focused to get optimized solar cell structure.

In this research paper presented some simulated results of variety of grating structure, the higher order diffraction angle with the transmission of light, study of the light reflection, and uncovers the mechanism at the back light extraction from the grating structures. The mechanism of the light trapping interaction (guided mode) among the front and back gratings is analyzed, which is considerably increases the light trapping by $154 \%$ compare to the reference solar cell. This

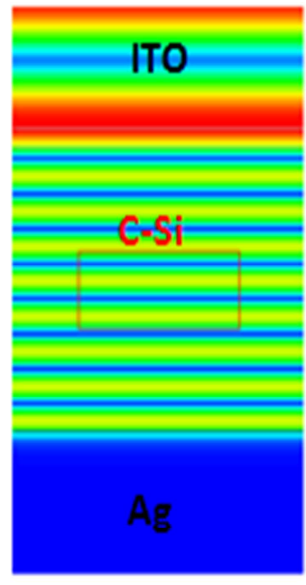

(a)

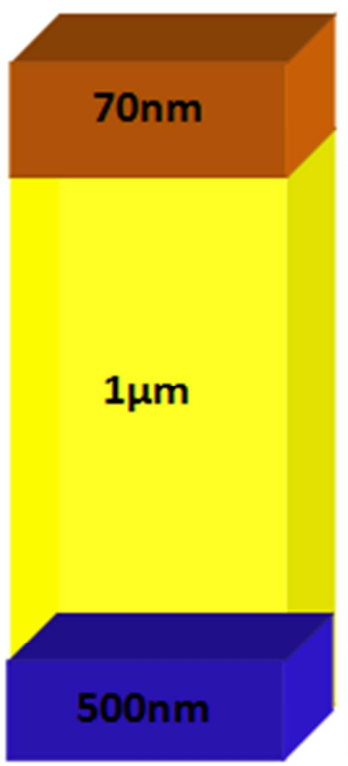

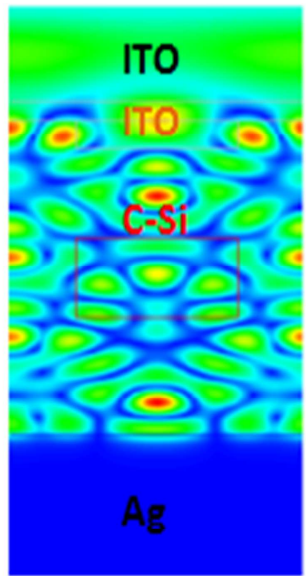

(b)

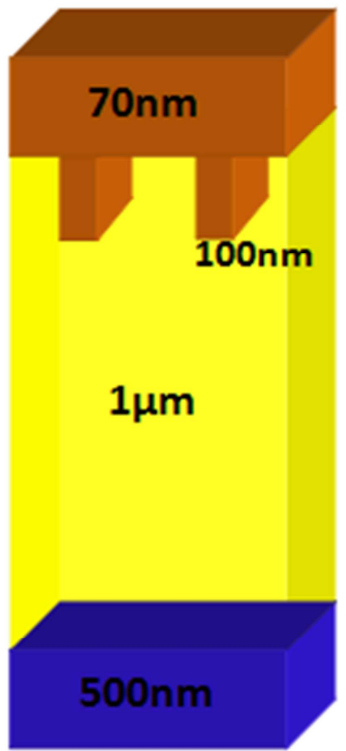

simulation results demonstrate that the designed structure given improved light harvestings. The optimizations of the designed metal and dielectric based solar structures are more contributed to the significant outcome of short circuit current, absorption and quantum efficiency.

\section{Designing Approach}

\subsection{Different Crystalline Silicon Thin Film Solar Cells}

The optical design for light trapping in ultrathin film silicon solar cells is developed by using RCWA method.

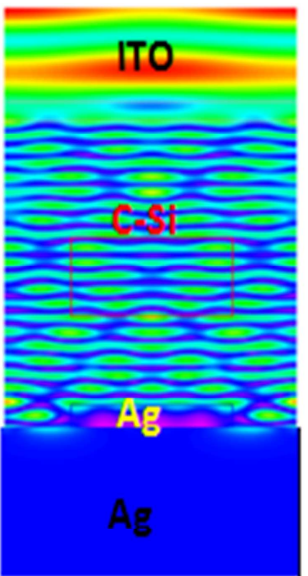

(c)

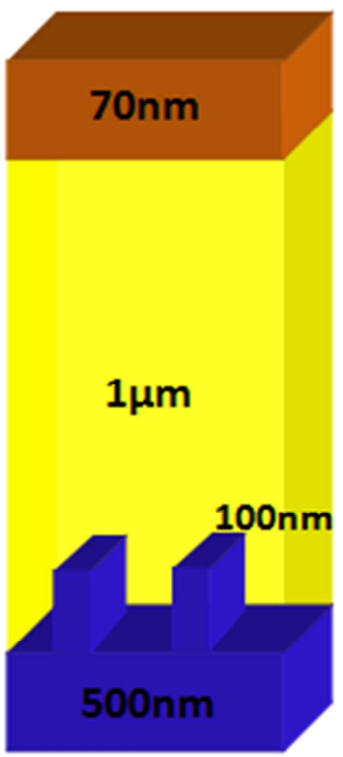

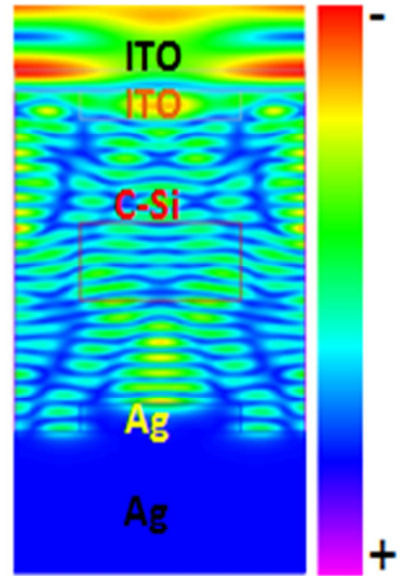

(d)

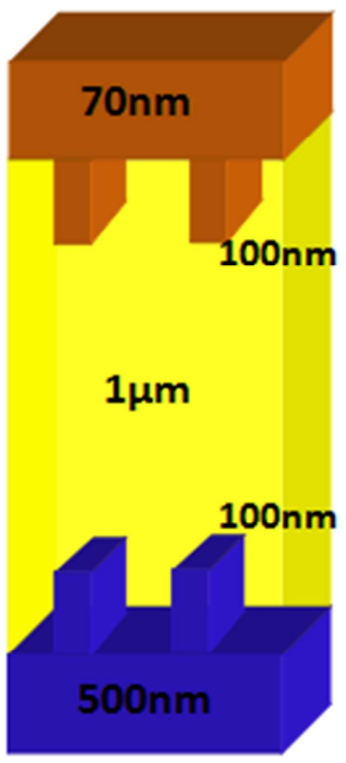

Figure 1. The electric field distributions of various thin film solar cells (a) reference (b) metal substrate with single ITO grating (c) metal substrate with single Ag grating (d) planar structure with dual grating. The thickness of the different periodic layer is shown through corresponding schematic diagram.

This calculation is particularly useful for complicated structures (Bio-sensor, LED, Solar cells) also known as simple and past method. It can solve Maxwell's equations with boundary (periodic and radiating) conditions [8]. The designing solar cell structure consists of anti-reflection coating (ARC), periodic metal and dielectric GRA. Here, 70 $\mathrm{nm}$ thicknesses of ITO deposited on c-Si and serve as transparent conducting oxide as well antireflection coating (ITO) layer [9]. The crystalline silicon layer acts as active layer and silver is a perfect back reflector. This geometric structure is fully comfortable with ARC layer to enhance the capture of incident light and the reflection losses are 
minimized. The crystalline silicon (c-Si) active region consist of $1 \mu \mathrm{m}$ with gratings $(100 \mathrm{~nm}$ thick) are separately embedded. These gratings are kept in a particular position (period) and compared with metal back reflector and planar structure. The performance of solar cell is primarily depends on grating structures only. Those grating based structure includes only bottom $\mathrm{Ag}$ grating and dual grating (Ag and ITO) cell structure. The thin film solar cells light management is highly trained by implementing light trapping techniques that are based on the use of special layers are called metal back reflectors (or substrate). These metal (Ag) substrate thickness is $500 \mathrm{~nm}$ and it has located underneath the grating structure.

\subsection{Schematic Diagram with TE Field Distribution}

In figure 1 represent the electric field of the different solar cell with suitable schematic diagram. Figure 1 (a-d) shows the electric field analysis of two way electric field analysis. The thin film crystalline silicon solar cell with metal back reflector as represented in figure 1 (a), the transmitted light passing through into absorber layer and reflected back due to Ag substrate (reference cell). It is called as double path length. When the ITO top grating integrated with reference solar cell, the incident light reflected back again as show in figure 1 (b). The metal back reflector performs as a perfect mirror. In figure 1 (c) the electric field interaction is improved well for the reason of that front ITO and back Ag grating. The top of the active region dielectric grating embedded, so the electric field interaction increased via incident light spreading inside (active region) as shown figure 1 (b) $\&(\mathrm{~d})$. The gratings $\left(\mathrm{T}_{\mathrm{g}}=100 \mathrm{~nm}\right)$ are played important role to increase the optical path length and life time of the light particles. In figure 1 (d) shows top (Si/ITO) and bottom $(\mathrm{Si} / \mathrm{Ag})$ grating used to enhance light trapping mechanism for the improvement of short-circuit current (Jsc) and cell efficiency $(\eta)$. This typical electric field distribution shows the effect of scattering, diffraction (optical) properties and it has been depends on the size and shape of the gratings. Among these four designed devices, efficient scattering and guiding light modes can be observed. This mechanism of guiding light gives increased residing of light which ultimately improves the absorption in silicon active region.

\section{Result and Discussion}

\subsection{Absorption Spectra Comparison of TE \& TM Polarization}

In this work, studied different silicon thin film solar cells named as 'A' cell (ARC+Substrate), 'B' cell (ARC+Top GRA+Substrate), 'C'cell (ARC+Bottom GRA + Substrate) and 'D'cell (ARC+Dual GRA+Substrate). This type of thin film solar cell provides a optimized system where guided/Bloch mode engineering plays significant performance to amplify the overall absorption. The absorption characteristics of the different solar cells are calculate as shown in figure $2(\mathrm{a} \& \mathrm{~b})$ under both (TE/TM) polarization modes. The first cell 'A' has less light trapping (green) due to the absence of back reflector. Further, the periodic incorporation done subsequently like substrate, top ITO, bottom Ag and dual grating. This addition makes remarkable enhancement within the active region due to that noticed absorption development.
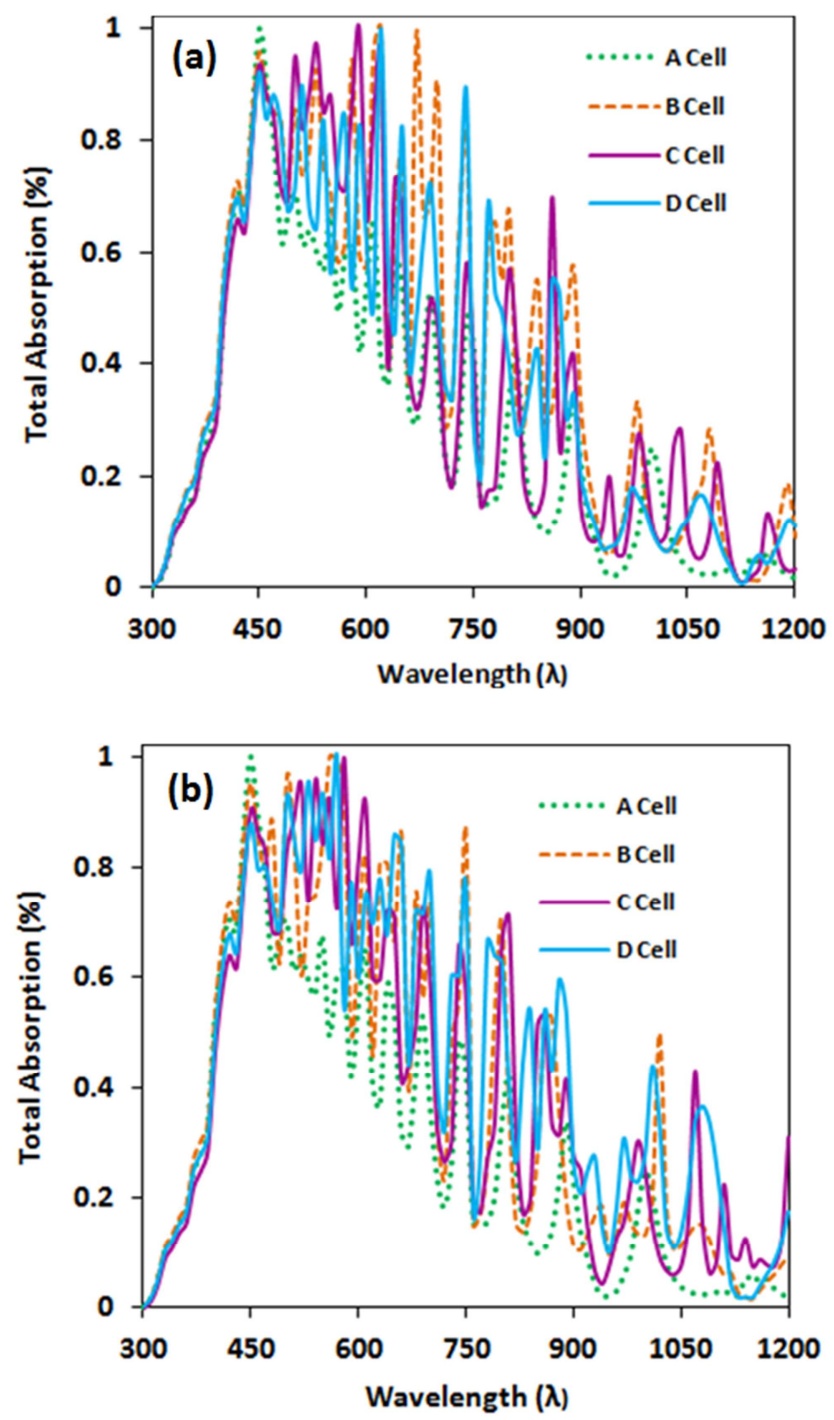

Figure 2. Absorption spectra of thin film solar cells $A, B, C$ and $D$ (a) TE, (b) $T M$.

Overall, cell ' $D$ ' showed better optical performance with respect to dual grating. The dielectric and metal gratings are extending the light path length within the crystalline silicon active layer by large diffraction angle. The diffraction angle calculated by using followed equation,

$$
n_{0} \sin \theta_{m}+n_{i} \sin \theta_{i}=\frac{m \lambda}{d}
$$

where, $n_{i}$ is the refractive index of the incident material and $\mathrm{n}_{\mathrm{o}}$ is the outgoing material refractive index, $\mathrm{d}$ is the constant, $\mathrm{m}$ is the diffractive order $(0,1,-1,2,-2 \ldots), \theta_{\mathrm{i}}$ and $\theta_{\mathrm{m}}$ is the incident and diffractive angle [10]. The strong absorption appeared from 450 to $900 \mathrm{~nm}$, the sharp peaks are appeared 
by Fabry-Perot interference. The TM field absorption curve enhanced well with the reason of surface plasmon polariton (SPP), field excitation and guided mode resonance. Figure 2 (b) shows significant collection of the photon absorption proved with field distribution and intensity profile (figure 4).

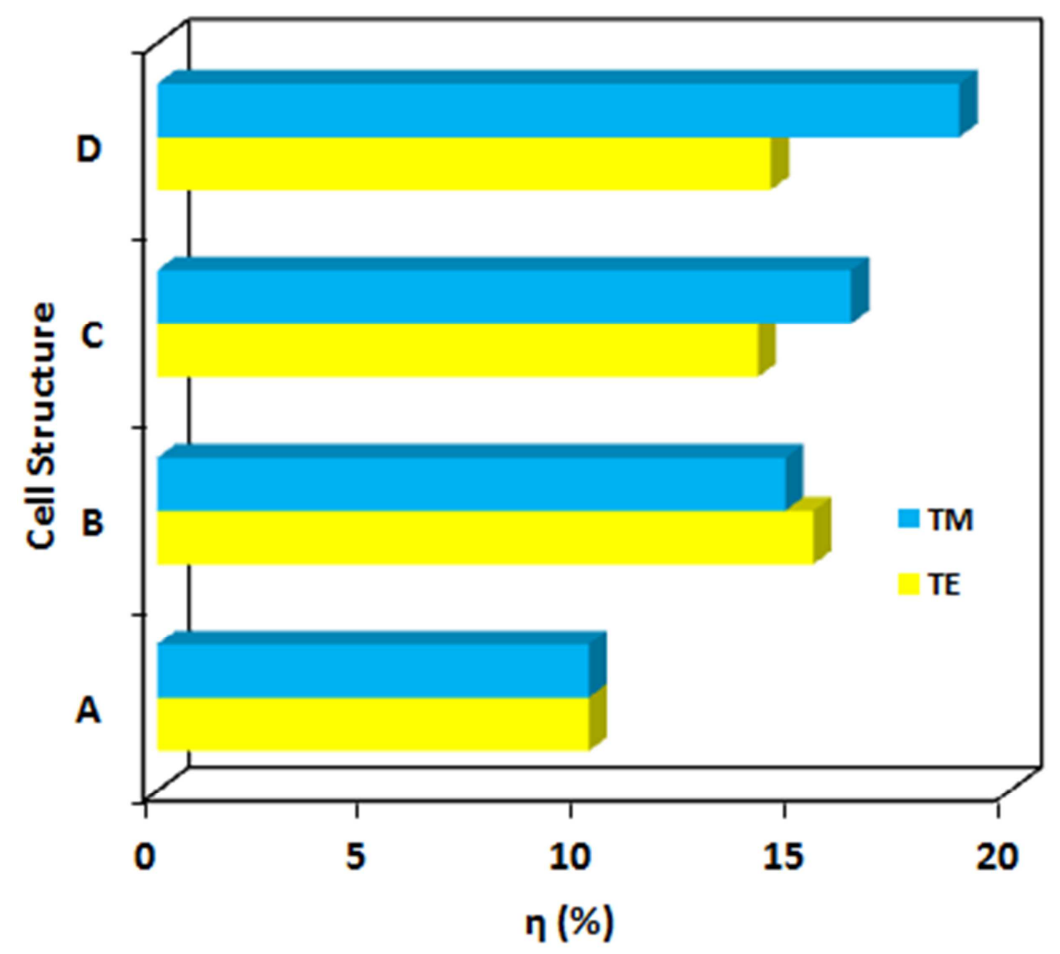

Figure 3. The cell efficiency ( $\eta$ ) of different thin film solar cell structures.

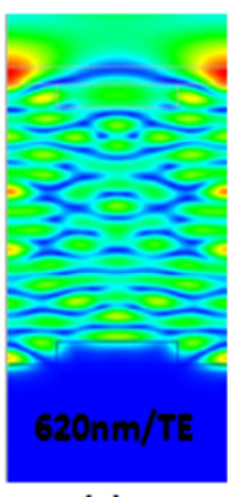

(a)

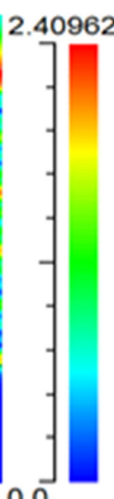

0.0

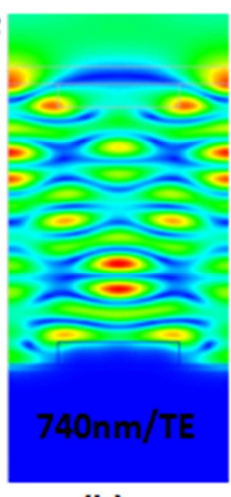

(b)

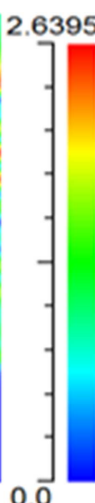

0.0

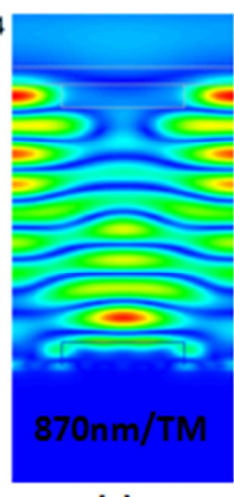

(c)

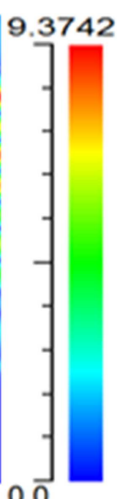

$\overrightarrow{0}$

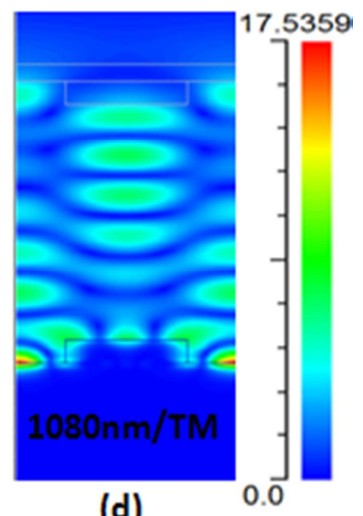

(d)

Figure 4. The transverse electric (TE) and magnetic field (TM) distribution at different incident wavelength.

\subsection{Cell Efficiency and Field Distribution (TE/TM)}

Figure 3 shows the cell efficiency of different crystalline silicon thin film solar cells at both polarization conditions. Here, the back reflector plays pivotal role to improve the collection of the photons. Solar cell 'A' depicts the less efficiency due to without back reflector. As includes back reflector/substrate enhanced cell performance as shown in Cell 'B'. Similarly, single dielectric and metal grating cell (cell $\mathrm{C}$ and $\mathrm{D}$ ) has shows enhanced optical performance. At last, combined both dielectric (top) and metal (bottom) gratings and have noticed remarkable cell efficiency at TE $(\sim 14.4 \%)$ and TM (18.82\%) modes. Surface plasmon is the electrons charges in which perform coherent fluctuations on the silver boundary layers and also localized at the normal direction within the Thomas-Fermi surface length [11]. It can notice the strong field intense at the top of the $\mathrm{Ag}$ grating at $620 \mathrm{~nm}$ and $740 \mathrm{~nm}$ as shows in figure 4 (a)\&(b) due to Fabry-Perot (FP) resonance. The enhanced electric field with guided mode resonance (GMR) can be observed in the UVVisible region. Here, red and cyan colors are represents maximum electric field strength and guided modes. The transverse magnetic field distribution at longer wavelengths (870 \& 1080nm) is shown in figure 4 (c \& d). The rectangular metal grating structure can scatter more incident light and shows plasmonic enhancement at metal boundaries specially corners [13]. The plasmon oscillations (quantum of plasma) are propagating within the metal nanostructure at longitude direction with the amplitude $\left(\Delta \mathrm{n}_{\max }\right)$. The quanta of 
volume plasmons having energy $(\sim 10 \mathrm{eV})$,

$$
\hbar \omega_{p}=\hbar \sqrt{\frac{n e^{2}}{m_{0} \varepsilon_{0}}}
$$

where, eigen frequency $\left(\omega_{\mathrm{p}}\right)$, the electron density $(n)$. The maximum number of electrons participates during in this oscillation and the incidence light can propagate along the metal surface with a broad spectrum [14]. From the field intensity profile, metal and dielectric interface associated with strong surface plasmon resonance (SPR) and skin effect as shown in figure 4 (c) \& (d). The skin effect/depth $(\delta)$ depends on the magnetic field (TM) and frequency $(\omega)$ of the conducting layer. Due to plasmonic effect, the field propagation enhanced considerably at IR spectral (870nm) region. At wavelength $1080 \mathrm{~nm}$ the field distribution remarkably elevated though SPR. Silver materials having 52 $\mathrm{nm}$ bulk mean free path and the skin depth lies between 24$29 \mathrm{~nm}$ from the wavelength range of 300-1200nm [Bohren and Huffman, 1983].

\section{Conclusions}

In conclusion, we have investigated the embedded ITO and Ag grating within thin c-Si absorber for efficient solar cell design. The performance of top and bottom grating structure with an ultra thin metal film was properly chosen as reflector. The planar c-Si thin film with single/dual grating ultrathin film plasmonic solar cells were compared and discussed by using RCWA method. In first, c-Si is deposited on metal substrate and the bottom grating structure is defined and metal is deposited to form a c-Si-Ag metal grating. After creating the first grating is bonded to a back reflector. Finally, ITO is deposited on the top of the c-Si layer and to form a cSi-ITO grating. In this paper focused on specific material system of silicon and the effect of single and double grating layers in plasmonic solar cells. The light absorbance and cell efficiency $(\sim 14.4 \%, 18.82 \%)$ are increased in dual grating thin film solar cells and proved through field distributions. These results are promising way for the realization of high efficiency thin film solar cells.

\section{References}

[1] M. A. Green, "Lambertian, light trapping in textured solar cells and light-emitting diodes: Analytical solutions," Prog. Photovoltaics: Research and Applications, vol. 10, no. 4, pp. 235-241, April, 2002.
[2] M. L. Hossain, W. Qarony, M. K. Hossain, M. K. Debnath, M. J. Uddin, and Y. H. Tsang, "Effect of back reflectors on photon absorption in thin-film amorphous silicon solar cells," Appl Nanosci, vol. 7, no. 7, pp. 489-497, August 2017.

[3] L. Zhao, Y. H. Zuo, C. L. Zhao, H. L. Li, and H. W. Diao, “A highly efficient light- trapping structure for thin film silicon solar cells," Sol Energy, vol. 84, no. 1, pp. 110-115, January 2010 .

[4] M. F. Nezhad, N. Shahtahmassebi and M. Behdani, "Improvement efficiency of thin-film solar cell by plasmonic properties of silver," Optik, vol. 127, pp. 111110-1-3, May 2016.

[5] S. Saravanan, and R. S. Dubey, "Optical absorption enhancement in 40nm ultrathin film silicon solar cells assisted by photonic and plasmonic modes," Opt. Commun, vol. 377, pp. 65-69, May 2016.

[6] J. Zhang, Z. Yu, Y. Liu, H. Chai, J. Hao, and H. Ye, "Dual interface gratings design for absorption enhancement in thin crystalline silicon solar cells," Opt. Commun, vol. 399, pp. 6267, September 2017.

[7] K. V. Sreekanth, R. Siddharhan, and V. M. Murukeshan "Gap modes assitsted enhanced broadband light absorption in plasmonic thin film solar cell," J. Appl. Phy., Vol. 110, 033107-1-5, August 2011.

[8] M. Paulsen, L. T. Neustock, S. Jahns, J. Adam, and M. Gerken, "Simulation methods for multiperiodic and aperiodic nanostructured dielectric waveguides," Opt. Quant. Electron, vol. 49, pp. 107-1-14, January 2017.

[9] X. Guo, J. Liu, and S. Zhang, "Design of light trapping structure for ultrathin Solar cells," Photonics and Optoelectronics (P\&O), vol. 3, pp. 66-69, March 2014.

[10] G. Zheng, W. Zhang, L. Xu, Y. Chen, and Y. Liu, "Absorbance enhancement of thin film solar cells with front double dielectric and back metallic grating," Infrared Phys Techn, vol. 67, pp. 52-57, July 2014.

[11] N. Das, "Nanostructured Solar Cells," InTech, Web of Science, 312, February 2017.

[12] S. Lee, and S. Kim "Optical absorption characteristic in thin aSi film embedded between an ultrathin metal grating and a metal reflector," IEEE Photonics J, vol. 5, no. 5, pp. 4800610 1-10, October 2013.

[13] Y. Yin, Z. Yu, Y. Liu, H. Ye, W. Zhang, Q. Cui, X. Yu, P. Wang, and Y. Zhang, "Design of plasmonic solar cells combining dual interface nanostructure for broadband absorption enhancement," Opt. Commun, vol. 333, pp. 213218, July 2014.

[14] H. Raether, "Excitation of plasmons and interband transitions by electrons," Springer Tr Mod Phys, vol. 88, pp. 1-180, January 2006. 